\title{
One-Stage Bilateral Total Hip Arthroplasty for Hemophilic Spontaneous Ankylotic Hip: A Case Report
}

\author{
Mariko Asahi1 ${ }^{*}$, Atsushi Kusaba1, Akihiko Maeda², Koji Kanzaki², Saiji Kondo', Takahide Tsuchiya ${ }^{3}$ \\ ${ }^{1}$ Institute of Joint Replacement and Rheumatology, Ebina General Hospital, Kawaraguchi, Ebina, Kanagawa, Japan \\ ${ }^{2}$ Department of Orthopaedic Surgery, Showa University Fujigaoka Hospital, Fujigaoka, Aoba-ku, Yokohama, Japan \\ ${ }^{3}$ Department of Hematology, Ebina General Hospital, Kawaraguchi, Ebina, Kanagawa, Japan \\ Email: *ma1ri2ko2000@yahoo.co.jp
}

How to cite this paper: Asahi, M., Kusaba, A., Maeda, A., Kanzaki, K., Kondo, S. and Tsuchiya, T. (2020) One-Stage Bilateral Total Hip Arthroplasty for Hemophilic Spontaneous Ankylotic Hip: A Case Report. Case Reports in Clinical Medicine, 9, 269-274.

https://doi.org/10.4236/crcm.2020.99038

Received: August 2, 2020

Accepted: September 12, 2020

Published: September 15, 2020

Copyright (c) 2020 by author(s) and Scientific Research Publishing Inc. This work is licensed under the Creative Commons Attribution International License (CC BY 4.0).

http://creativecommons.org/licenses/by/4.0/

\begin{abstract}
Total hip arthroplastys (THAs) for hemophilic spontaneous ankylotic hips are rare. We performed one-stage bilateral THA for hemophilic patient and I would like to report the results of the case. A 61-year-old male had been diagnosed with mild hemophilia $\mathrm{A}$ as a neonate. He had severe pain in both hips and moderate pain in both knees. His hips were ankylotic and had no mobility. Uncemented simultaneous bilateral THAs were performed under general anesthesia. This case suggests that one-stage THA can be a good option for hemophilic hips, provided that sufficient substitution therapy is used.
\end{abstract}

\section{Keywords}

Hemophilic Arthropathy, One-Stage Bilateral THA, Complication

\section{Introduction}

Hemophilic arthropathy often involves bilateral joints [1]. Total hip arthroplasty (THA) is an appropriate procedure for disabling hemophilic arthropathy, despite the higher risks of bleeding and the concern of complications in surgery due to poor bone quality and poor joint mobility [2]. The first reported hip arthroplasty in a patient with hemophilia was in 1967 [3], and subsequently the fixation methods of components (i.e., cemented or uncemented) have been controversial for hemophilic hips [4]. Only one case of one-stage bilateral THA for hemophilic coxa-arthropathy has been described [2]. Here, we report the case of a patient with hemophilia A who was treated with one-stage bilateral THA. The patient and his family were informed that data from the case would be submitted 
for publication, and gave their consent.

\section{Case Presentation}

A 61-year-old male had been diagnosed with mild hemophilia A as a neonate. His two younger brothers had also been diagnosed with hemophilia A and one of them had died due to bleeding. The patient had been receiving factor VIII substitution treatment three times each week. After his retirement, he consulted us due to difficulty in sitting in a chair. He had severe pain in both hips and moderate pain in both knees. His hips were ankylotic and had no mobility (Table 1). He could walk aided by a crutch, but only very slowly. A radiographic examination showed end-stage destruction of both hip and knee joints (Figure 1). At the first examination, the serum factor VIII level was $5 \%$ and inhibitor of factor VIII was not present. HCV and HIV were negative. To relieve the joint dysfunction and pain in the hip joints, simultaneous bilateral THA was planned. The knees joints also appeared to require arthroplasties, but these procedures seemed to be difficult prior to hip mobilization by THA.

Under the guidance of a hematologist, $800 \mathrm{~mL}$ of autologous blood was preserved before surgery. Uncemented simultaneous bilateral THAs were performed under general anesthesia, with a blood salvage system used during surgery. The procedure was performed in a half lateral position via a Watson-Jones approach. Surgery was started on the right side with the option of postponing work on the other side if excessive bleeding occurred during the initial procedure. Blood loss on the right side was $450 \mathrm{~mL}$, which was less than expected, and thus we continued with the procedure on the left side. This procedure was performed identically to that on the right side. Blood loss on the left side was 730 $\mathrm{mL}$, giving a total loss of $1180 \mathrm{~mL}$. The total time for completion of the bilateral procedure was $4 \mathrm{~h}$.

Both acetabular and femoral implants were uncemented. The cup was a Spongiosa Metal II (GHE, ESKA Orthodynamics GmbH, Lübeck, Germany), the stem was an SL PLUS (Smith \& Nephew AG, Rotkreuz, Switzerland), and the bearing couple was a $28-\mathrm{mm}$ ceramic on conventional polyethylene (Figure 2). Bilateral extensive soft tissue release and adductor tenotomy were necessary to mobilize

Table 1. Motion range in hips.

\begin{tabular}{ccccc}
\hline & Pre-Op. & \multicolumn{3}{c}{ Post Op. 3 years } \\
& Right & Left & Right & Left \\
\hline Flexion & 45 & 20 & 50 & 45 \\
Extension & -45 & -20 & -15 & -10 \\
Abduction & -10 & 10 & 10 & 15 \\
Adduction & 10 & -10 & 20 & 25 \\
External Rotation & -15 & 15 & 0 & 0 \\
Internal Rotation & 15 & -15 & 20 & 25 \\
\hline
\end{tabular}




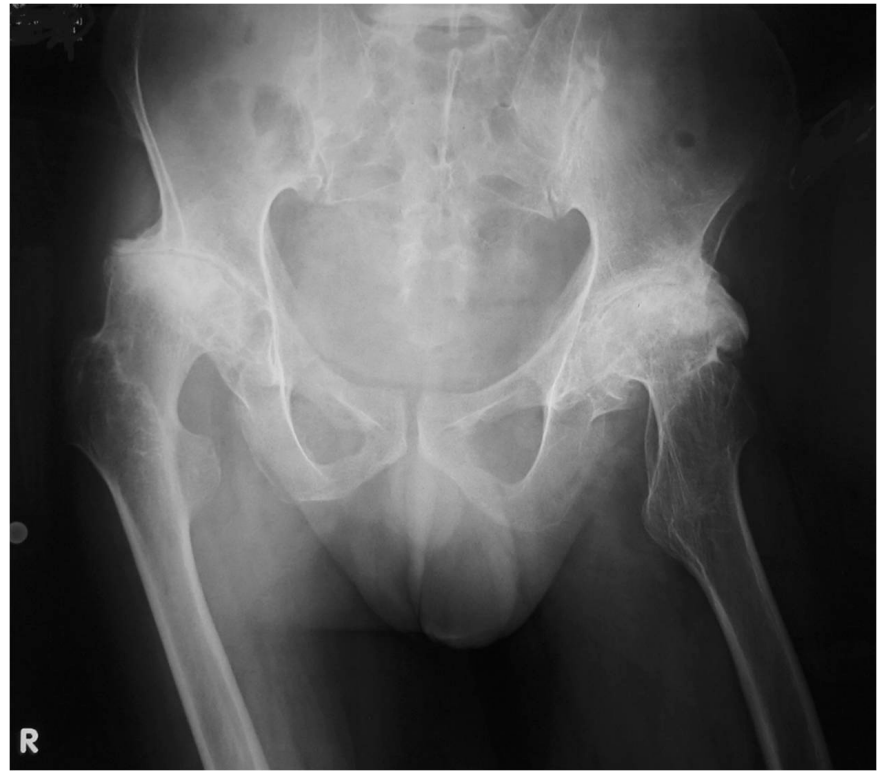

Figure 1. Radiography showing end-stage destruction of the hip and knee joints.

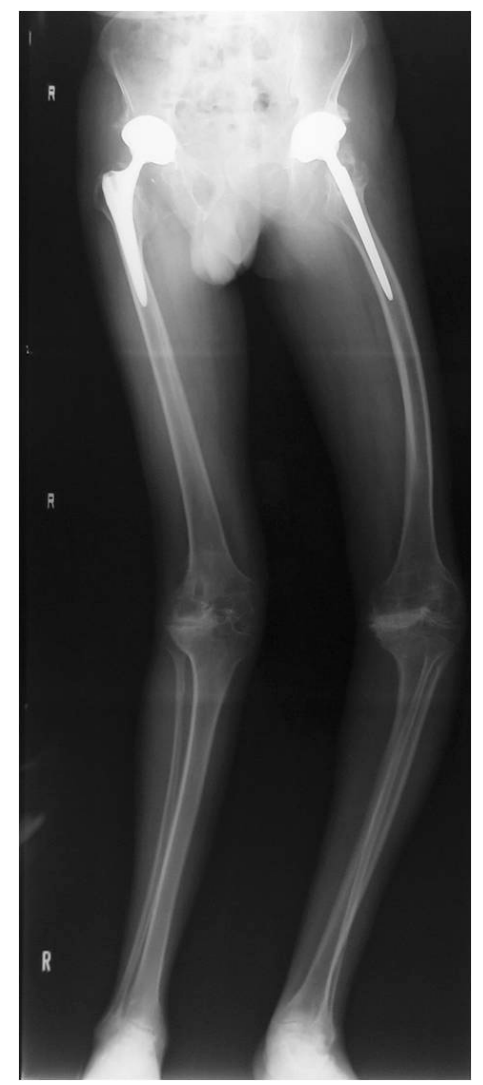

Figure 2. The implant used in surgery.

the ankylotic hips: bilateral adductor origin tenotomy, right adductors insertion release from the femur, right iliopsoas tenotomy, bilateral quadriceps and sartorius origin release, and right gluteus maximums insertion release. Trochanteric fracture occurred bilaterally, and the fractures were repaired by fixing the frag- 
ment sutures on the stem holes. Substitution treatment was planned based on the guidelines of the Japanese Society for Thrombosis and Hemostasis. But, one hour postoperatively, $3000 \mathrm{U}$ of Freeze-dried Human Blood Coagulation Factor VIII (Confact F, Astellas Pharma Inc., Tokyo, Japan) was substituted for wound bleeding and prolonged APTT time, and wound hemostasis was obtained. Factor VIII (3000 U, $50 \mathrm{U} / \mathrm{kg}$ ) was substituted at 8-hour intervals after surgery until post-operative day (POD) 11, every $12 \mathrm{~h}$ until POD 16 days, and every $24 \mathrm{~h}$ until POD 25, after which the regular prophylactic dose (1000 U three times a week) of factor VIII was administrated twice a week. The total dose of factor VIII after surgery was 142,500 U for 25 days. During surgery, $800 \mathrm{~mL}$ of autologous blood was transfused and $300 \mathrm{~mL}$ of salvaged blood was reinfused. Red cell concentrate in mannitol adenine phosphate was transfused just after surgery $(560 \mathrm{~mL})$ and on POD $1(280 \mathrm{~mL})$ to compensate for anemia. There were no wound problems.

Gait exercise started two days after surgery with full weight bearing. The patient was discharged from hospital on POD 44. His hospitalization was 1.5 times longer than that of non-hemophilic patients with one-stage THAs. At one year follow-up, the trochanteric fractures had united and no loosening had occurred. He obtained mobility in both hips, can sit on a chair easily, and can walk with single cane support (Table 1). He is free from hip joint pain, but still has moderate knee joint pain. He is fully satisfied with the result.

He died two years later after the THA.

\section{Discussion}

In the only previous report of one-stage bilateral THA in a patient with hemophilia, Lofqvist et al. performed one-stage cemented THAs for a patient with mild hemophilia [2]. The procedure resulted in excessive intraoperative blood loss of $2.9 \mathrm{~L}$ that required a total factor VIII of $144,000 \mathrm{U}$. At final follow-up, the patient complained of pain during walking and at rest, and had a markedly reduced walking ability. Aseptic loosening occurred bilaterally (bilateral cup-loosening after 53 months, and bilateral stem-loosening after 66 months) and the patient died of AIDs in 1986 [2].

From 1972 to 1990, Kelley et al. [4] performed 26 cemented and 6 uncemented THAs for 32 hemophilic patients. Three late infections occurred in the cemented arthroplasties and required reoperation. Aseptic loosening occurred in $21 \%$ of the cemented stems and $23 \%$ of the cemented cups, whereas neither infection nor loosening occurred in the uncemented components [4]. These results led to the use of uncemented components for hemophilic hips, even though the follow-up period for the uncemented arthroplasty was only three years and that for the cemented procedure was eight years [4].

Simultaneous vs. two stage bilateral THA in non-hemophilic hips continues to evoke controversy [5]. Aghayer et al. suggested that one-stage bilateral THA is equally safe or safer than a two-stage intervention from the perspective of intra- 
and post-operative complications [6]. However, Berend et al. argued against simultaneous bilateral THA because of a higher rate of postoperative inpatient complications and adverse events in patients who underwent this procedure under a single anesthetic [5]. Eggli et al. reported better functional outcomes after one-stage THA, especially for very stiff hips with a preoperative range of motion $<50$ [7]. In a meta-analysis, Haverkamp et al. found no difference in the prevalence of complications related to bleeding between one- and two-stage THA for non-hemophilic hip lesions [8].

For non-hemophilic hips, we have performed 363 pairs of one-stage bilateral THA and obtained excellent results. Therefore, we performed one-stage bilateral THA for the patient in the current case, although, as mentioned above, we initially planned to perform only the right side procedure depending on the blood loss during this procedure. The cost for one-stage THAs may be a problem depending on the country [8], but the cost had no influence on our preoperative plan. However, we used additional substitution therapy because of bleeding after the operation. We planned to use factor VIII for 3 weeks postoperatively, and we anticipated that the required total factor VIII would be 126,000 U. We ultimately used factor VIII for 44 days after the operation, and the total used was 188,500 $\mathrm{U}$. This resulted in a financial cost to our hospital because the factor VIII dosage was above the limit of the insurance coverage. Nelson et al. used tranexamic acid, a fibrinolysis inhibitor, pre- and post-operatively to minimize the factor VIII requirement, along with postoperative use of a hip spica for 3 weeks to minimize the risk of bleeding through immobilization, which also reduced the requirement for factor VIII [9]. Lofqvist et al. also gave tranexamic acid at 6- to 8-hour intervals for about 7 days to suppress local fibrinolysis [2].

In our patient, bilateral TKA was also necessary for severe pain in the knees, but we were unable to complete these procedures because the patient died two years later after the THA. His wife did not want to talk about the cause of death, but she said that he was very happy with the THA outcome. Therefore, this case suggests that one-stage THA can be a good option for hemophilic hips, provided that sufficient substitution therapy is used.

\section{Conclusion}

THAs for hemophilic patients are rare, therefore there are few papers, and the technique for operation and perioperative management has not been established. This case suggests that one-stage THA can be a good option for hemophilic hips, provided that sufficient substitution therapy is used. And for the hemophilic patient who hopes to have surgery, I desire that the researches for hemophilic patient make progress and the financial relief will rise up in the near future.

\section{Conflicts of Interest}

The authors declare no conflicts of interest regarding the publication of this paper. 


\section{References}

[1] Luck Jr., J.V., Silva, M., Rodriguez-Merchan, E.C., Ghalambor, N., Zahiri, C.A. and Finn, R.S. (2004) Hemophilic Arthropathy. Journal of the American Academy of Orthopaedic Surgeons, 12, 234-245. https://doi.org/10.5435/00124635-200407000-00004

[2] Löfqvist, T., Sanzén, L., Petersson, C. and Nilsson, I.M. (1996) Total Hip Replacement in Patients with Hemophilia. 13 Hips in 11 Patients Followed for 1 - 16 Years. Acta Orthopaedica Scandinavica, 67, 321-324. https://doi.org/10.3109/17453679609002323

[3] Habermann, B., Eberhardt, C., Hovy, L., Zichner, L., Scharrer, I. and Kurth, A.A. (2007) Total Hip Replacement in Patients with Severe Bleeding Disorders. A 30 Years Single Center Experience. International Orthopaedics, 31, 17. https://doi.org/10.1007/s00264-006-0136-5

[4] Kelley, S.S., Lachiewicz, P.F., Gilbert, M.S., Bolander, M.E. and Jankiewicz, J.J. (1995) Hip Arthroplasty in Hemophilic Arthropathy. The Journal of Bone and Joint Surgery, 77, 828-834. https://doi.org/10.2106/00004623-199506000-00003

[5] Berend, K.R., Lombardi Jr., A.V. and Adams, J.B. (2007) Simultaneous vs. Staged Cementless Bilateral Total Hip Arthroplasty: Perioperative Risk Comparison. The Journal of Arthroplasty, 22, 111-115. https://doi.org/10.1016/j.arth.2007.03.043

[6] Aghayev, E., Beck, A., Staub, L.P., Dietrich, D., Melloh, M., Orljanski, W. and Röder, C. (2010) Simultaneous Bilateral Hip Replacement Reveals Superior Outcome and Fewer Complications Than Two-Stage Procedures: A Prospective Study Including 1819 Patients and 5801 Follow-Ups from a Total Joint Replacement Registry. BMC Musculoskeletal Disorders, 11, Article No. 245. https://doi.org/10.1186/1471-2474-11-245

[7] Eggli, S., Huckell, C.B. and Ganz, R. (1996) Bilateral Total Hip Arthroplasty: One Stage versus Two Stage Procedure. Clinical Orthopaedics and Related Research, 328, 108-118. https://doi.org/10.1097/00003086-199607000-00019

[8] Haverkamp, D., van den Bekerom, M.P., Harmse, I. and Schafroth, M.U. (2010) One Stage Bilateral Total Hip Arthroplasty, Is It Safe? A Meta-Analysis. HIP International, 20, 440-446. https://doi.org/10.1177/112070001002000405

[9] Nelson, I.W., Sivamurugan, S., Latham, P.D., Matthews, J. and Bulstrode, C.J. (1992) Total Hip Arthroplasty for Hemophilic Arthropathy. Clinical Orthopaedics and Related Research, 276, 210-213. https://doi.org/10.1097/00003086-199203000-00028 